\title{
An Orbital Angular Momentum Spectrometer for Electrons
}

Tyler R. Harvey ${ }^{1}$, Vincenzo Grillo ${ }^{1,2,3}$, Benjamin J. McMorran ${ }^{1}$

${ }^{1 .}$ Department of Physics, University of Oregon, Eugene, OR 97405, USA

2. CNR-Istituto Nanoscienze, Centro S3, Via G. Campi 213/a, I-41125 Modena, Italy

${ }^{3}$ CNR-IMEM Parco Area delle Scienze 37/A, I-43124 Parma, Italy

Measurement of magnetization with atomic resolution via dichroic electron energy loss spectroscopy (EELS) with vortex beams remains a hotly-discussed and as-yet unproven goal for electron microscopy [1-5]. A satisfactory explanation for the lack of successful demonstrations of this technique has not yet emerged. However, a number of recent predictions $[2,5,6]$ for dichroism include one common oversight: post-selection of a final state without orbital angular momentum (OAM) is not automatically guaranteed in a real dichroic EELS experiment.

Unlike dichroic absorption spectroscopy experiments with circularly polarized light, where the final photon state is a no-photon state which necessarily has zero angular momentum, electrons in a transmission electron microscope are not absorbed, and instead pass through a specimen. Incident electron vortices with $m_{l} \hbar$ OAM have a non-zero probability to scatter to an outgoing state with unchanged $m_{l} \hbar$ OAM. Dichroic electron energy loss spectroscopy, then, must involve some postselection for the component of the final state which has nonzero $\Delta m_{l}$. There exist qualitative methods to post-select for more of this component of the final state [7], and semi-quantitative interferometric methods [8] that do not work for the inelastically scattered final states which are incoherent with respect to the initial state that one must measure in a dichroic EELS experiment. In order to accomplish dichroic EELS, a quantitative non-interferometric method to spatially separate OAM will be necessary.

We propose a magnetic field-based mechanism for on-axis separation of electron OAM modes. Inside a magnetic lens field approximated by the Glaser model [9], with the addition of the lowest-order radial curvature, we can write the vector potential as

$$
\boldsymbol{A}=\frac{B_{0} \rho}{2\left(1+\frac{z^{2}}{R^{2}}\right)}\left(1-\frac{\rho^{2}}{2 b^{2}}\right) \hat{\phi}
$$

where $B_{0}$ is the field strength at the center of the lens, R sets the longitudinal extent of the field, and $\rho=$ $\left(x^{2}+y^{2}\right)^{1 / 2}$ is the radial position coordinate. The length parameter $b$ defines the length scale of radial curvature. The Hamiltonian which results from the addition of the vector potential show in (1) includes, among other terms, two parabolic lensing terms: the standard Glaser lensing term, and additional term which depends on OAM.

$$
\Delta H_{\text {lens }}=\left(\frac{e^{2} B_{0}^{2}}{8 m_{e}\left(1+\frac{z^{2}}{R^{2}}\right)}-\frac{e B_{0} L_{z}}{4 m_{e} b^{2}\left(1+\frac{z^{2}}{R^{2}}\right)}\right) \rho^{2}
$$

Figures 1 and 2 show multislice-simulated [10] phase structure of vortices in the back focal plane of a lens which includes only the second, OAM-dependent lens term. The simulation parameters are chosen to exaggerate the effect for illustration purposes and are not physically reasonable.

If we ignore the effect of higher-order aberrations produced by the vector potential in (1) and calculate the shift of the focal length of a thin magnetic lens due only to the OAM-dependent lensing, we see 


$$
\Delta f_{m_{l}}=f_{0} \frac{2 L_{z}}{e B_{0} b^{2}}
$$

where $f_{0}$ is the standard focal length of a thin magnetic lens [9] due to the first term in the Hamiltonian in (2). We expect that, under suitable conditions, this change of focal length can be used, in combination with a round aperture, to preferentially pass a single focused OAM mode. This spectrometer disperses by orbital angular momentum along the z-axis. Figure 3 shows simulated far-field intensities of the vortices shown in Figures 1 and 2 after interaction with the OAM-dependent lens.

A major challenge in physical implementation of this mechanism will be to maximize the difference in focal length between modes that differ by one quantum of OAM. The unitless quantity $\frac{\hbar}{e B_{0} b^{2}}$ is typically very small in standard magnetic lenses. To approach unity, one must either decrease the field strength such that the focal length becomes impractically large or decrease the curvature length scale below a reasonable size for machined lens components.

If successfully implemented, the OAM measurement device we propose here will allow for near-perfect post-selection of OAM modes and therefore will enable far more reliable dichroic EELS experiments.

References:

[1] J. Verbeeck et al., Nature 467 (2010) p.301.

[2] S. Lloyd et al., Phys. Rev. Lett. 108 (2012) p.074802.

[3] P. Schattschneider et al., Phys. Rev. Lett. 110 (2013) p.189501 .

[4] P. Schattschneider et al., Ultramicroscopy 136 (2014) p.81-85.

[5] J. Rusz and S. Bhowmick, Phys. Rev. Lett. 111 (2013) p.105504.

[6] J. Rusz et al., Phys. Rev. Lett. 113 (2014) p.145501.

[7] K. Saitoh et al., Phys. Rev. Lett. 111 (2013) p.074801.

[8] L. Clark et al., Phys. Rev. A 89 (2014) p.053818.

[9] L. Reimer and H. Kohl, "Transmission Electron Microscopy: Physics of Image Formation", (Springer, New York), p.22.

[10] V. Grillo et al., New Journal Phys 15 (2013) 093026.

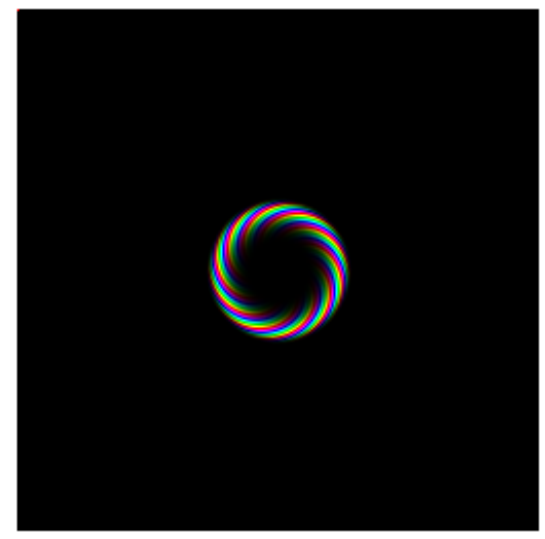

Figure 1: Simulated phase and intensity of $+10 \hbar$ vortex subject to the OAM-dependent lens term with $b=3$ um, $B_{0}=$ $0.01 \mathrm{~T}, R=10 \mathrm{um}$, and beam enerav $\mathrm{E}=100 \mathrm{keV}$.

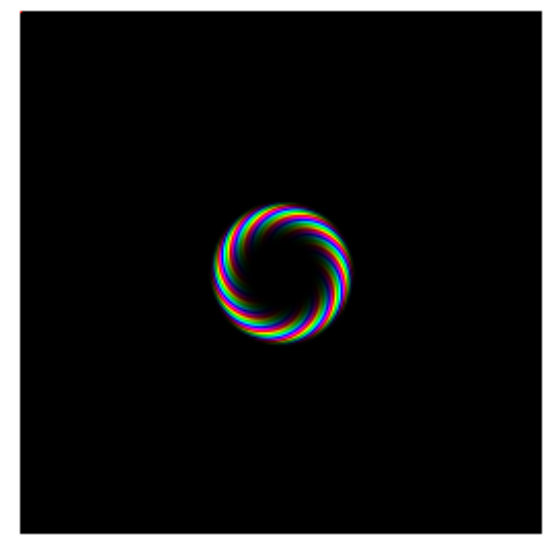

Figure 2: Simulated phase and intensity of $-10 \hbar$ vortex under the same conditions.

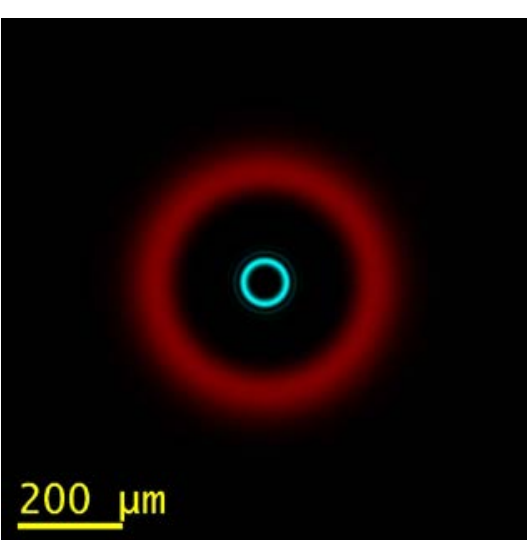

Figure 3: $+10 \hbar$ (small, teal) and $-10 \hbar$ (large, red) vortices after propagation over $100 \mathrm{~m}$ after passing through the spectrometer field. Scale applies to all imaqes. 